\title{
Circular RNA ciRs-126 promotes hypoxia/ reoxygenation cardiac injury possibly through miR-21
}

\author{
Changming $\operatorname{Tan}^{1 *}$, Jianming $\mathrm{Li}^{1}$, Zhaoshun Yuan ${ }^{1}$ and Yongxin $\mathrm{Mu}^{2}$
}

\begin{abstract}
Background: This study aimed to analyze the role of circular RNA ciRs-126 in hypoxia/reoxygenation cardiac injury (H/R).

Methods: Expression of ciRs-126 and miR-21 in plasma samples from patients with $\mathrm{H} / \mathrm{R}$ and healthy controls was determined by RT-qPCR. Correlations were analyzed by linear regression. Overexpression of ciRs-126 and miR-21 was achieved in cardiomyocytes to explore their crosstalk. The roles of ciRs-126 and miR-21 in H/R-induced apoptosis of cardiomyocytes were analyzed using cell apoptosis assay.

Results: CiRs-126 was upregulated and miR-21 was downregulated in H/R patients. They were inversely correlated across plasma samples from H/R patients. In H/R cardiomyocytes, ciRs-126 was upregulated and miR-21 was downregulated. In cardiomyocytes, ciRs-126 overexpression decreased miR-21 level and reduced the inhibitory effects of miR-21 overexpression on $\mathrm{H} /$ R-induced cell apoptosis.
\end{abstract}

Conclusions: Circular RNA ciRs-126 may suppress miR-21 expression to promote H/R cardiac injury.

Keywords: Hypoxia/reoxygenation cardiac injury, ciRs-126, miR-21, Apoptosis

\section{Background}

Hypoxia/reoxygenation-induced cardiac injury (H/R), also known as myocardial ischemia or cardiac ischemia, is caused by reduced blood flow [1]. H/R is usually caused by atherosclerosis, blood clot, and coronary artery spasm [2]. With the development of $H / R$, heart attack, arrhythmia, and heart failure may occur, leading to a high mortality rate $[3,4]$. In severe cases, $15 \%$ of patients with $\mathrm{H} / \mathrm{R}$ will die before hospitalization, and $15 \%$ will die during hospitalization. Even worse, about 10\% of survivors will die within one year after discharge $[5,6]$. $\mathrm{H} / \mathrm{R}$ is usually treated with angioplasty or bypass surgery [7], while injuries are usually irreversible. Therefore, novel treatment approaches are still needed.

\footnotetext{
* Correspondence: tanchangming79@csu.edu.cn

'Department of Cardiovascular Surgery, The Second Xiangya Hospital, Central South University, No.139 Middle Renmin Road, 410011 Changsha, Hunan, China

Full list of author information is available at the end of the article
}

Previous studies of $H / R$ have identified considerable molecular factors involved in the initiation and development of $\mathrm{H} / \mathrm{R}$ [8-10]. Increased understanding of the molecular mechanism of $\mathrm{H} / \mathrm{R}$ facilitates the development of novel treatment approaches $[9,10]$. Circular RNA (circRNA) is a class of circular RNAs with 3' and 5' ends covalently closed [11]. Previous studies have identified critical functions of circRNA in diverse pathological and physiological processes, including $H / R$ [12]. A recent study has shown that circRNA ciRs- 126 promotes acute kidney injury [13], while its role in $H / R$ is unknown. We performed a preliminary deep sequencing analysis and observed the altered expression of ciRs-126 in H/R and its inverse correlation with miR-21, which plays protective roles in $H / R$ [14]. This study was therefore carried out to analyze the crosstalk between ciRs-126 and miR21 in $\mathrm{H} / \mathrm{R}$.

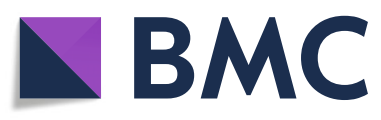

(c) The Author(s). 2021 Open Access This article is licensed under a Creative Commons Attribution 4.0 International License, which permits use, sharing, adaptation, distribution and reproduction in any medium or format, as long as you give appropriate credit to the original author(s) and the source, provide a link to the Creative Commons licence, and indicate if changes were made. The images or other third party material in this article are included in the article's Creative Commons licence, unless indicated otherwise in a credit line to the material. If material is not included in the article's Creative Commons licence and your intended use is not permitted by statutory regulation or exceeds the permitted use, you will need to obtain permission directly from the copyright holder. To view a copy of this licence, visit http://creativecommons.org/licenses/by/4.0/. The Creative Commons Public Domain Dedication waiver (http://creativecommons.org/publicdomain/zero/1.0/) applies to the data made available in this article, unless otherwise stated in a credit line to the data. 


\section{Methods}

\section{Patients and controls}

This study included a total of 60 patients with H/R (H/R group, 30 males and 30 females) and 60 healthy controls (30 males and 30 females) at The Second Xiangya Hospital, Central South University from May 2019 to May 2020. All participants were at the age of 38 to 66 years, with an average of $52.1 \pm 6.7$ years for patients and $52.0 \pm$ 6.8 years for controls. All patients were diagnosed based on the ACC/AHA/ESC/WHF practice guidelines. Table 1 lists patients' detailed clinical information. $H / R$ in the 60 patients was caused by the formation of blood clots in arteries. The patients were divided into 5 stage groups according to the time after infarction, namely, $<4 \mathrm{~h}, 4$ $6 \mathrm{~h}, 6-8 \mathrm{~h}, 8-10 \mathrm{~h}$, and 10-12 h. The number of H/R patients in each group was $11,12,11,16$, and 10 , respectively. The patients with chest pain of more than $12 \mathrm{~h}$ and those with cardiac injury caused by factors other than $H / R$ were excluded. All healthy controls received systemic physiological exams, and all physiological functions were within normal range. Patients with other severe diseases, such as cancers and diabetes, were excluded. This study was approved by the Ethics Committee of the Second Xiangya Hospital, Central South University. All patients and controls signed informed consent.

\section{Plasma and cells}

To exclude the effect of dietary on ciRs-126 and miR-21 levels, blood $(3 \mathrm{ml})$ was extracted into EDTA tubes from all patients and controls after they were fasted for more than $8 \mathrm{~h}$ and prepared as plasma samples by centrifugation for $15 \mathrm{~min}$ at $1200 \mathrm{~g}$. The plasma samples were kept in liquid nitrogen prior to use.

Primary cardiomyocytes (C-12,810, Sigma-Aldrich, USA) were cultured in Myocyte Growth Medium (Sigma-Aldrich) in an incubator with $5 \% \mathrm{CO}_{2}$ at $37{ }^{\circ} \mathrm{C}$ following the instructions provided by the vendor. Cells at passages 3 to 5 were used for all experiments.

\section{Cell transfections}

A vector expressing ciRs-126 was constructed using pcDNA3.1(+) CircRNA Mini Vector (Addgene) as the backbone. MiR-21 mimic and negative control (NC)

Table 1 Clinical parameters of the study subjects

\begin{tabular}{|c|c|c|c|}
\hline Clinical parameters & $\mathrm{H} / \mathrm{R}(n=60)$ & Healthy subjects $(n=60)$ & $P$ \\
\hline Sex (male/female) & $30 / 30$ & $30 / 30$ & 1.000 \\
\hline Age (years) & $52.1 \pm 6.7$ & $52.0 \pm 6.8$ & 0.643 \\
\hline Tobacco & $72 \%(43)$ & $58 \%(35)$ & 0.091 \\
\hline Hypertension & $76 \%(46)$ & 0 & $\neq$ \\
\hline Hypercholesterolemia & $77 \%(46)$ & 0 & $\neq$ \\
\hline Diabetes mellitus & $31 \%(19)$ & 0 & $\neq$ \\
\hline Body mass index (kg/m2) & $23 \pm 2,2$ & $22 \pm 1.8$ & 0.000 \\
\hline Previous Ml & $6 \%(4)$ & 0 & $\neq$ \\
\hline Previous CABG & $1.6 \%(1)$ & 0 & $\neq$ \\
\hline Previous PTCA & 0 & 0 & \\
\hline Glucose (mmol/L) & $3.1 \pm 1.1$ & $3.5 \pm 0.2$ & 0.000 \\
\hline High-sensitivity C-reactive protein (mg/L) & $15.13 \pm 6.66$ & $1.89 \pm 1.36$ & 0.000 \\
\hline $\mathrm{LDH}(\mathrm{U} / \mathrm{L})$ & $481.60 \pm 55.73$ & $157.71 \pm 22.94$ & 0.000 \\
\hline CK-MB (U/L) & $118.29 \pm 55.63$ & $10.11 \pm 2.16$ & 0.000 \\
\hline cTnT $(\mu \mathrm{g} / \mathrm{L})$ & $21.44 \pm 5.95$ & 0 & $\neq$ \\
\hline Aspirin use & $56 \%(34)$ & 0 & $\neq$ \\
\hline Clopidogrel use & $42 \%(25)$ & 0 & $\neq$ \\
\hline Beta blocker use & $84 \%(50)$ & 0 & $\neq$ \\
\hline Nitrates use & $77 \%(46)$ & 0 & $\neq$ \\
\hline Angiotensin-converting-enzyme inhibitor drugs use & $30 \%(18)$ & 0 & $\neq$ \\
\hline Angiotensin receptor blocker use & $11 \%(7)$ & 0 & $\neq$ \\
\hline Calcium channel blocker use & $9 \%(5)$ & 0 & $\neq$ \\
\hline Lipid-lowering drugs & $68 \%$ & 0 & $\neq$ \\
\hline
\end{tabular}


miRNAs were purchased from Sigma-Aldrich (USA). Cardiomyocytes were transfected with $40 \mathrm{nM}$ miRNA or $1 \mu \mathrm{g}$ expression vector using Lipofectamine 2000 (Invitrogen). To perform NC experiments, cells were transfected with empty vector or NC miRNA. Control (C) cells were untransfected cells. Cells were incubated with transfection mixtures for $6 \mathrm{~h}$ and in fresh medium for $48 \mathrm{~h}$ prior to the subsequent assays.

\section{$\mathrm{H} / \mathrm{R}$ model}

After transfections (including $\mathrm{C}$ and $\mathrm{NC}$ groups), cardiomyocytes were cultured under hypoxic conditions (1\% $\mathrm{O}_{2}, 95 \% \mathrm{~N}_{2}$ and $5 \% \mathrm{CO}_{2}$ ) for $2 \mathrm{~h}$ at $37{ }^{\circ} \mathrm{C}$ and in normoxic culture medium for $3 \mathrm{~h}$ at $37{ }^{\circ} \mathrm{C}$ to achieve reoxygenation.

\section{RNA preparations}

RNAs from plasma and cardiomyocytes were extracted using RNAzol (Sigma-Aldrich). After being treated with DNase I (Invitrogen) for $2 \mathrm{~h}$ at $37^{\circ} \mathrm{C}$ to remove genomic DNAs, RNA purity and integrity were examined by OD260/280 ratios and 6\% urea-PAGE.

\section{RT-qPCRs}

To synthesize cDNA samples, RNA samples with satisfactory quality were subjected to reverse transcriptions (RTs) using SS-III-RT system (Invitrogen). CiRs-126 expression was determined by qPCRs using SYBR Green Master Mix (Bio-Rad) with GAPDH, $18 \mathrm{~S}$ rRNA, or $\beta$ actin as the internal controls. Mature miR-21 level was determined using All-in-One ${ }^{\text {rat }}$ miRNA qRT-PCR reagent kit (GeneCopoeia). To perform the reverse transcription effectively, addition of poly (A) to mature miRNAs was performed (polyA tailing method), followed by miRNA RTs and qPCRs using RPL30 and $18 \mathrm{~S}$ rRNA as the internal controls. All steps were completed following the manufacturer's instructions. It is worth noting similar results were obtained using different internal endogenous controls. QPCRs were performed at $95^{\circ} \mathrm{C}$ for $1 \mathrm{~min}$ followed by 40 cycles of $10 \mathrm{~s}$ at $95^{\circ} \mathrm{C}$ and $48 \mathrm{~s}$ at $58^{\circ} \mathrm{C}$.

All qPCRs were performed with three technical replicates. Ct values of target genes were used normalized to the internal controls using the $2^{-\Delta \Delta C t}$ method. Supplemental Table 1 lists all primer sequences.

\section{Dual-luciferase assays}

Dual-GloR Luciferase Assay System (Promega, USA) was used to conduct the dual-luciferase assays. In brief, miR21 promoter was amplified using primer pair $\mathrm{Fr}$ TGTAAAACGACGGCCAGT and Re-CAGGAAACAG CTATGACC and cloned into the pmirGLO vector. Cardiomyocytes were transfected with the reporter plasmid and with/without ciRs-126. After 2 days, cells were washed, digested with trypsin, and collected by centrifugation for $3 \mathrm{~min}$ at $5000 \mathrm{~g}$. The cellular proteins were then extracted using $100 \mu \mathrm{L}$ of lysis buffer. $50 \mu \mathrm{L}$ of protein supernatants were added to a 96-well measuring plate and mixed in turn with $50 \mu \mathrm{L}$ of Luciferase Reagent and Stop \& Glo Reagent. The firefly luciferase activities were measured on Centro LB960 with renilla luciferase as the internal reference to correct the transfection efficiency.

\section{Western blot analysis}

Total proteins were extracted from transfected cells. After determining protein concentration using BCA Protein Assay Kit, the same amount of proteins were separated by SDS-PAGE and transferred onto membranes. Protein levels were detected using antibodies against caspase-3 (1:1000; sc-65,497), Bax (1:1000; sc-7480), Bcl2 (1:1000; sc-7382) and GAPDH (TA-09) (Yatai hengxin. Beijing, China), and horseradish peroxidase (HRP)-conjugated goat anti-rabbit IgG (1:1000; sc-2004, Santa Cruz, CA).

\section{Cell apoptosis assay}

Following cell transfections and H/R modeling, cardiomyocytes were collected, washed with ice-cold PBS, and resuspended in Annexin binding buffer to reach a final density of $10^{5}$ cells per ml (Dojindo). After that, cells were stained with Annexin V-FITC and PI (Dojindo) in the dark for $12 \mathrm{~min}$. Apoptotic cells were then analyzed by flow cytometry. In each experiment, three biological replicates were included.

\section{Statistical analysis}

Gene expression levels in plasma samples were expressed as average values of three technical replicates and compared using unpaired $t$ test. Data of three biological replicates of cell transfection groups were expressed as mean \pm SD values and compared by ANOVA Tukey's test. $P<0.05$ was considered statistically significant.

\section{Results}

\section{Altered ciRs-126 and miR-21 expressions were observed} in $\mathrm{H} / \mathrm{R}$

To determine the expression of ciRs-126 and miR-21 in H/ $\mathrm{R}$, plasma samples were collected from $H / R$ patients $(n=60)$ and controls $(n=60)$, and ciRs-126 and miR-21 expression were determination by RT-qPCR. Compared to the control group, ciRs-126 was significantly overexpressed in $\mathrm{H} / \mathrm{R}$ group (Fig. 1 A, $p<0.001$ ), while miR-21 was significantly underexpressed in $\mathrm{H} / \mathrm{R}$ group (Fig. $1 \mathrm{~B}, p<0.001$ ). The expression of ciRs-126 and miR-21 were positively and negatively correlated with different stages of $\mathrm{H} / \mathrm{R}$ patients, respectively (Fig. $1 \mathrm{C}$ and Fig. 1D, $p<0.05$ ). Furthermore, the correlation between the expression of Cir-126, CK-MB and cTnT in H/ $\mathrm{R}$ samples were also performed. The results indicate that the 
A

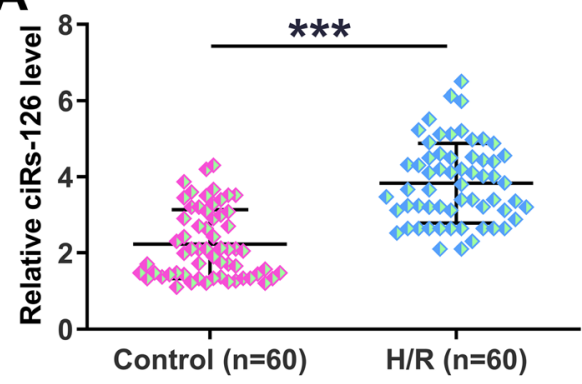

C

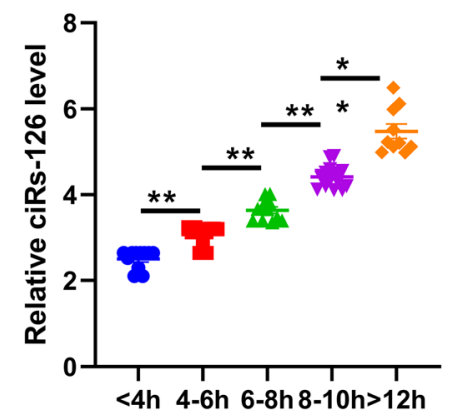

B

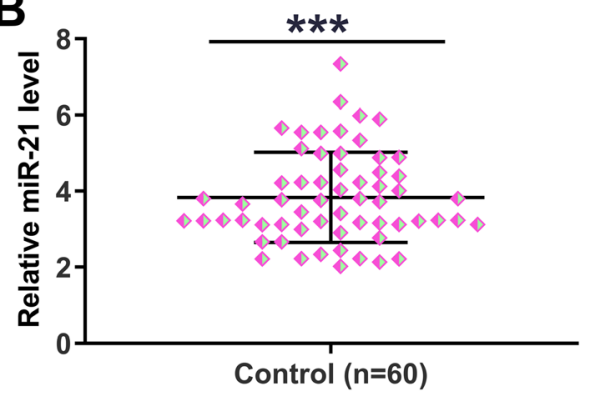

D

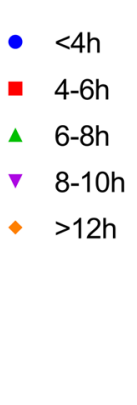

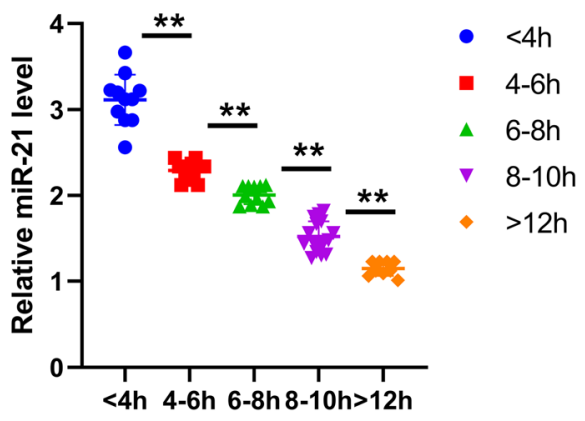

Fig. 1 Altered expression of ciRs-126 and miR-21 was observed in H/R. To determine the expression of ciRs-126 and miR-21 in H/R, plasma samples were collected from H/R patients ( $n=60)$ and controls $(n=60)$, followed by the determination of ciRs-126 (A) and miR-21 (B) expression by RT-qPCR. The expression of ciRs-126 and miR-21 in patients at different H/R stages were detailed in (C) and (D). Gene expression levels in plasma samples were expressed as average values of triplicate technical replicates. "1" indicated the related expression compared to GAPDH. **, $p<0.05 ;{ }^{* * *}, p<0.001$

expression of Cir-126 was correlated with the amount of CK-MB and $\mathrm{CTnT}$ (supplemental figure-1). Our data suggested that altered expression of ciRs-126 and miR-21 may be involved in $\mathrm{H} / \mathrm{R}$.

\section{A significant and inverse correlation between ciRs-126} and miR-21 was observed across plasma samples from $\mathrm{H}$ /

\section{$R$ patients}

Correlations between ciRs-126 and miR-21 across plasma samples from both $H / R$ patients and healthy controls were analyzed by linear regression. It was observed that ciRs-126 and miR-21 were inversely and significantly correlated with each other across plasma samples from $\mathrm{H} / \mathrm{R}$ patients (Fig. 2 A). However, no significant correlation between ciRs-126 and miR-21 was observed across plasma samples from the healthy controls (Fig. 2B). Therefore, ciRs-126 and miR-21 may interact with each other in $H / R$, and their crosstalk is likely mediated by certain $\mathrm{H} / \mathrm{R}$-related pathological factors.
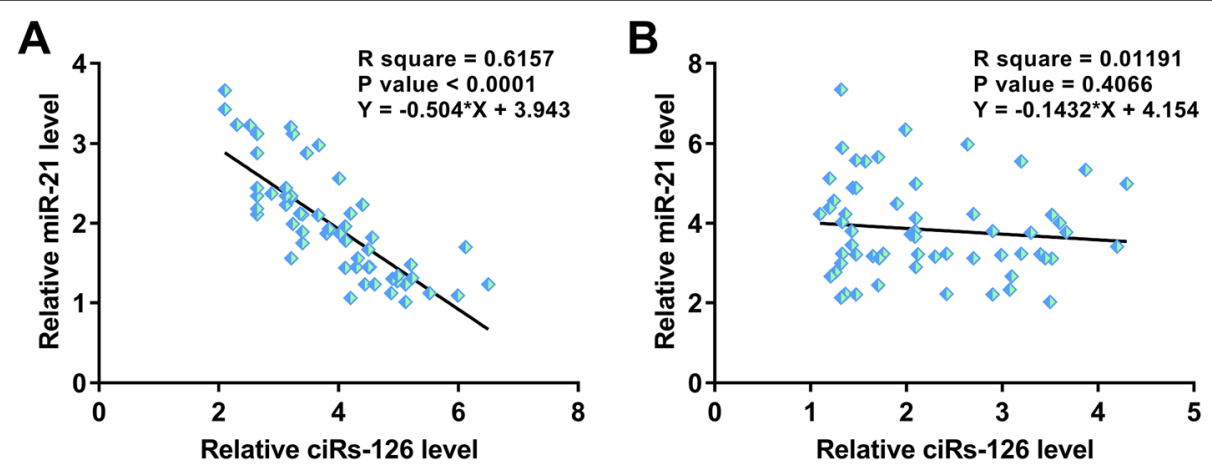

Fig. 2 A significant and inverse correlation between ciRs-126 and miR-21 was observed across plasma samples from H/R patients. Correlations between ciRs-126 and miR-21 across plasma samples from both H/R patients $(\mathbf{A})$ and healthy controls $(\mathbf{B})$ were analyzed by linear regression 
CiRs-126 overexpression decreased miR-21 expression in $\mathrm{H} / \mathrm{R}$ cell model but not in non $\mathrm{H} / \mathrm{R}$ treated cells

Our bioinformatics analysis and a previous study have suggested that ciRs-126 and miR-21 could only form an 8-base pairing. Hence it may not be sufficient for ciRs-126 to sponge miR-21. Luciferase assay was then performed to investigate the promoter activity of miR-21 influenced by ciRs-126 in cardiomyocytes and the results showed no difference between ciRs-126 transfection and control groups (Supplemental figure-2). To explore the crosstalk between ciRs-126 and miR-21, ciRs-126 expression vector or miR21 mimic was transfected into H/R-treated cardiomyocytes and non H/R treated cardiomyocytes. Overexpression of ciRs-126 and miR-21 was confirmed by RT-qPCR (Fig. $3 \mathrm{~A}, p<0.05$ ). It was found that ciRs-126 overexpression decreased miR-21 level in H/R cell model, but not in non $\mathrm{H} / \mathrm{R}$ treated cells (Fig. $3 \mathrm{~B}, p<0.05$ ). However, miR-21 overexpression failed to significantly alter ciRs-126 expression in both $\mathrm{H} / \mathrm{R}$-treated and untreated cardiomyocytes (Fig. 3 C). Therefore, ciRs-126 is likely a conditional dependent upstream inhibitor of miR-21 in H/R.
ciRs-126 overexpression attenuated the inhibitory effect of miR-21 on H/R-induced cardiomyocyte apoptosis

CiRs-126 and miR-21 levels in H/R-treated cardiomyocytes were measured by RT-qPCR. The results showed that $H / R$ cell model exhibited significantly overexpressed ciRs-126 (Fig. 4 A, $p<0.05$ ) and under-expressed miR-21 compared to untreated cells (Fig. $4 \mathrm{~B}, p<0.05$ ). Thus, the roles of ciRs-126 and miR-21 in regulating $H / R$-induced cardiomyocyte apoptosis was analyzed using cell apoptosis assay. CiRs-126 overexpression significantly increased cell apoptosis, while miR-21 overexpressed decreased cells apoptosis. In addition, ciRs-126 overexpression reduced the inhibitory effects of miR-21 overexpression on H/R-induced cell apoptosis and apoptosisrelated protein expression (Fig. 4 C-D, $p<0.05$ ).

\section{Discussion}

This study mainly investigated the involvement of ciRs126 and miR-21 in H/R and explored their crosstalk. We found that ciRs-126 was overexpressed in H/R. In addition, ciRs-126 could downregulate miR-21 to promote the apoptosis of cardiomyocytes induced by H/R.

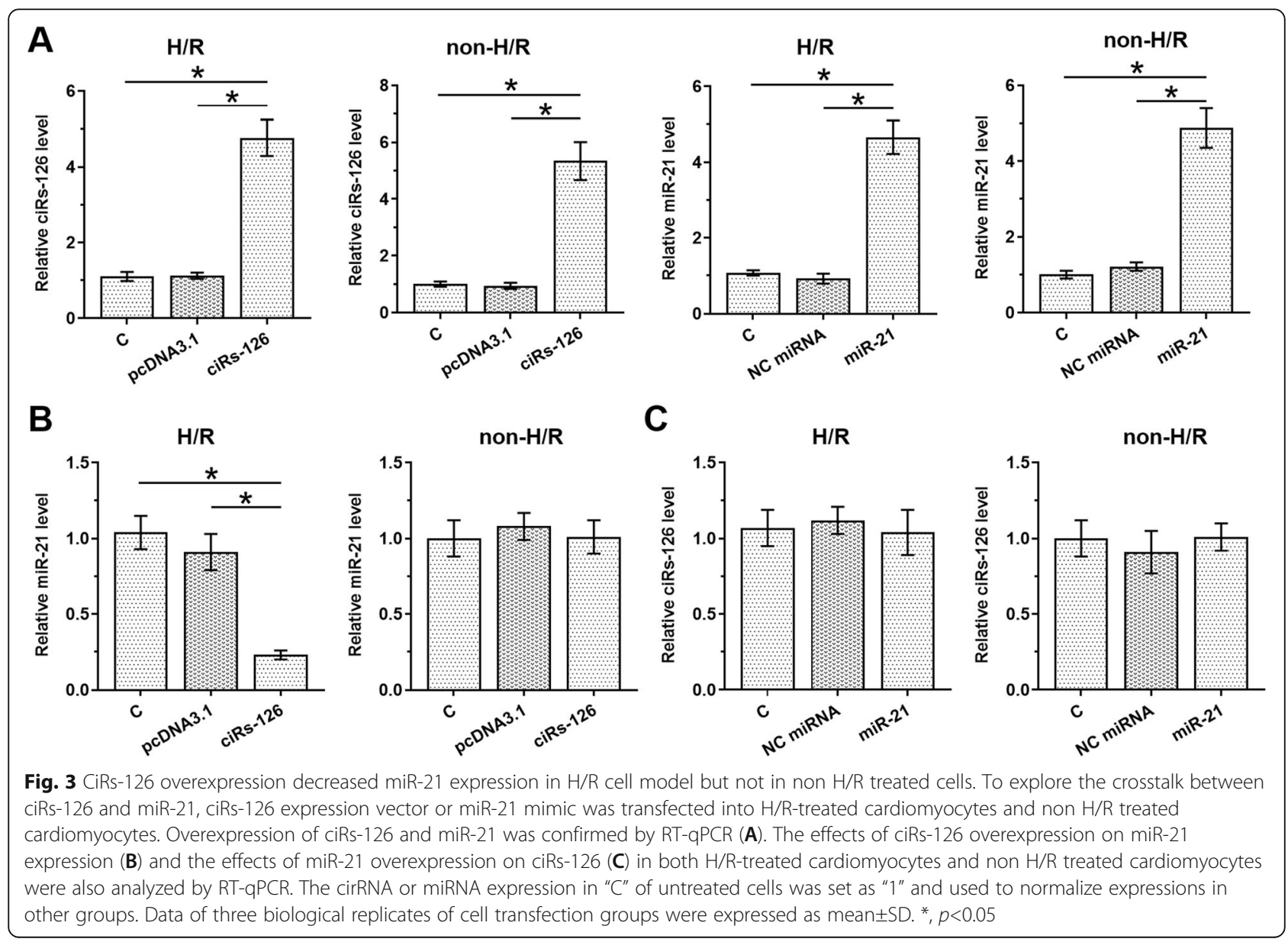



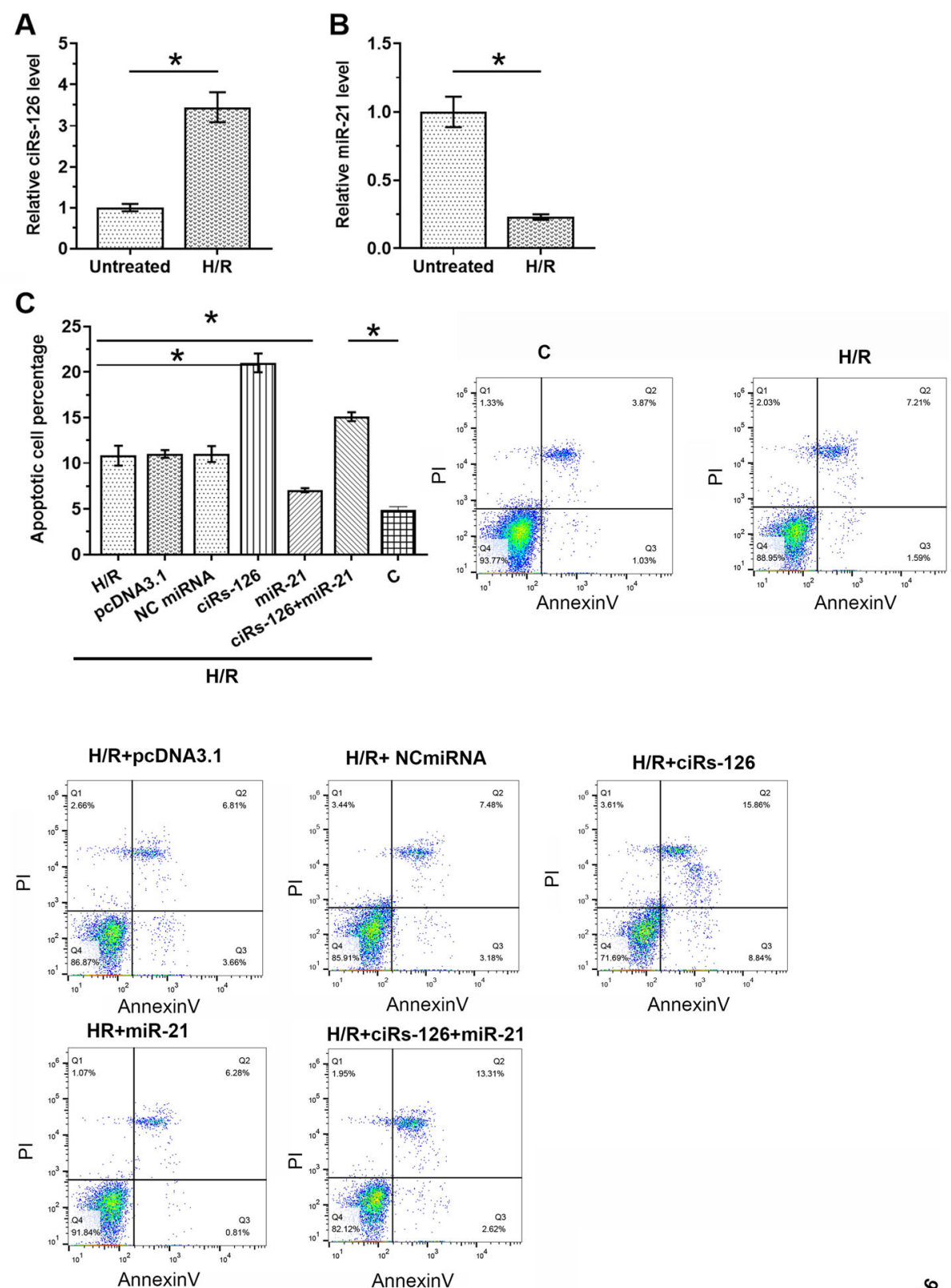

D

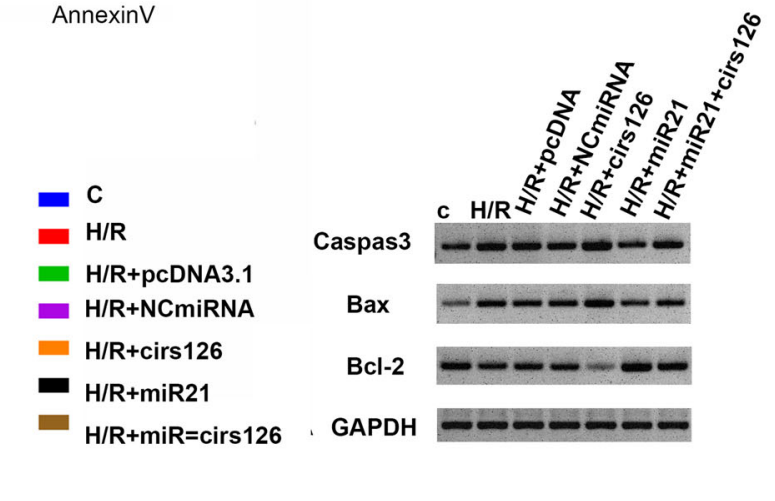

Fig. 4 (See legend on next page.) 
(See figure on previous page.)

Fig. 4 CiRs-126 overexpression attenuated the inhibitory effect of miR-21 on H/R-induced cardiomyocyte apoptosis. Cardiomyocytes were subjected to H/R treatment, followed by the measurement of expression levels of ciRs-126 (A) and miR-21 (B) by RT-qPCR. Cell apoptosis assay was performed to analyze the roles of ciRs-126 and miR-21 in regulating cardiomyocyte apoptosis induced by H/R (C). Western blot analysis for caspase 3, Bax, and Bcl-2 from cardiomyocytes induced by H/R (D). Data of three biological replicates of cell transfection groups were expressed as mean \pm SD. ${ }^{*}, p<0.05$

The function of ciRs-126 has only been studied in acute kidney injury and polycystic ovarian syndrome[13, 15]. It was observed that ciRs-126 was significantly overexpressed in patients with acute kidney injury and could sponge miR-126-5p to participate in disease progression[13]. In polycystic ovarian syndrome, ciRs-126 is downregulated and targets miR-21-PDCD4-ROS axis to suppress ovarian granulosa cell proliferation[15]. This study, for the first time, reported the upregulation of ciRs-126 in H/R. In addition, ciRs-126 overexpression significantly increased the apoptosis of cardiomyocytes induced by $H / R$. Therefore, ciRs- 126 may promote $H / R$ injuries by promoting cell apoptosis. Moreover, H/R treatment increased ciRs-126 expression level in cardiomyocytes. Therefore, H/R inducible ciRs-126 promotes cardiac injuries by increasing cell apoptosis.

Huang et al. recently showed that miR-21 is significantly under-expressed in H/R model and miR-21 overexpression suppressed cell apoptosis and autophagic activity in cardiac injury, possibly through AKT /mTOR pathway[14]. Consistently, our study observed the downregulation of miR-21 in patients with $H / R$ and cardiomyocytes after $H / R$ treatment[14]. In addition, miR-21 overexpression decreased the apoptosis of cardiomyocytes induced by $\mathrm{H} / \mathrm{R}$. Therefore, our study further confirmed the protective role of miR-21 in H/R.

It has been reported that ciRs- 126 could sponge miR21 to suppress the role of miR-21 in the proliferation and apoptosis of ovarian granulosa cell proliferation[15]. However, based on our bioinformatics analysis and this study, ciRs-126 and miR-21 can only form an 8-base pairing, which may not be sufficient for ciRs-126 to sponge miR-21. In this study, luciferase assay results showed that there was no direct regulation between miR-21 and ciRs- 126 . We showed that ciRs-126 could downregulate miR-21 in $H / R$ models but not in cells without $H / R$ treatment. Therefore, H/R-related factors may mediate the crosstalk between ciRs-126 and miR21 . The results indicated the existence of other conditional dependent regulation mechanisms for ciRs-126 and miR-2.Our result was consistent with previous in acute kidney injury cells study, namely, Circular RNA sponge of miR-126 (or ciRs-126) was most altered compared to healthy controls and disease controls[16]. No matter in the $H / R$ or acute kidney injury cells, the sponge between ciRs-126 and miR-21 was enhanced in the hypoxic cells. One likely explanation is that miR-126 has been shown to control hypoxia signaling and ciRs126 may be part of signaling cascades involving LRIG( the liner form of ciRs-126) and miR-126. The associated signaling pathways include EGFR family, tyrosineprotein kinase Met, and RET proto-oncogene[17, 18]. There may be many signaling cross-talks and feedback loops to regulate the homeostasis between ciRs-126 and miR-126. More detailed works need perform in future. Furthermore, the levels of ciRs-126 were also closly corelated to those of CK-MB and $\mathrm{CTnT}$, which indicate the dead/dying cardiomyocytes, showed that the ciRs-126 was also released from such cells and indicated the cell injury caused by hypoxia. Taken together, the conditional existence of ciRs-126/miR-21 in H/R cardiomyocytes indicates that ciRs-126 up-regulation and miR-21 down-regulation might be a pair of potential biomarkers for myocardial ischemia, especially at the early stage. More studies are needed in the future.

\section{Conclusions}

In conclusion, ciRs- 126 is overexpressed in $H / R$ and may downregulate miR-21 to promote the apoptosis of cardiomyocytes induced by $\mathrm{H} / \mathrm{R}$.

\section{Abbreviations}

H/R: Hypoxia/reoxygenation cardiac injury; circRNA: Circular RNA; RTs: Reverse transcriptions

\section{Supplementary information}

The online version contains supplementary material available at https://doi. org/10.1186/s12959-021-00355-x.

\section{Additional file 1.}

Additional file 2: Supplement Fig. 1. The correlation analysis between the expression of Cir-126, CK-MB and CTnT in H/R samples. Linear regression analysis results of the expression of $\mathrm{Cir}-126$ and the concentration of CK-MB (A)and CTnT(B) in H/R samples. The Pearson correlation coefficient is 0.910 and 0.905 , respectively.

Additional file 3: Supplement Fig. 2. Luciferase Assay. Luciferase activity detection by dual-luciferase reporter assay following cardiomyocytes treated with/without H/R. Cir-126 and reporter vector of miR-21 promoter were then transfected, the luciferase activity $(1.01 \pm 0.02 \mathrm{VS}$ $0.93 \pm 0.02 ; 10.84 \pm 0.07$ VS $0.16 \pm 0.01, p>0.5, d f=2)$ was then examined Students't test was performed to compare different groups. All data were presented as mean \pm SD in each figure. $n=3$.

\section{Acknowledgements}

Not applicable.

\section{Authors' contributions}

All authors contributed to the study conception and design. Material preparation, data collection and analysis were performed by CMT, JML, ZSY 
and YXM. The first draft of the manuscript was written by CMT, and all authors commented on previous versions of the manuscript. All authors have read and approved the final manuscript.

\section{Funding}

Not applicable.

\section{Availability of data and materials}

The datasets used and/or analyzed during the current study are available from the corresponding author on reasonable request.

\section{Declarations}

Ethics approval and consent to participate

This study was approved by the Ethics Committee of the Second Xiangya Hospital, Central South University. All the patients signed informed consent.

\section{Consent for publication}

Not applicable.

\section{Competing interests}

The authors declare that they have no competing interests.

\section{Author details}

'Department of Cardiovascular Surgery, The Second Xiangya Hospital, Central South University, No.139 Middle Renmin Road, 410011 Changsha, Hunan, China. ${ }^{2}$ Department of Medicine, University of California, La Jolla, CA, San Diego, USA.

Received: 19 May 2021 Accepted: 3 December 2021

Published online: 04 January 2022

\section{References}

1. Hausenloy DJ, Yellon DM. Myocardial ischemia-reperfusion injury: a neglected therapeutic target. J Clin Invest. 2013;123(1):92-100. doi: https:// doi.org/10.1172/JCl62874.

2. Kealey A. Coronary artery disease and myocardial infarction in pregnancy: a review of epidemiology, diagnosis, and medical and surgical management. Can J Cardiol. 2010;26(6):185-9. doi: https://doi.org/10.1016/s0828-282x(1 0)70397-4.

3. Kleinbongard P, Heusch G, Schulz R. TNFalpha in atherosclerosis, myocardial ischemia/reperfusion and heart failure. Pharmacol Ther. 2010;127(3):295-314. doi: https://doi.org/10.1016/j.pharmthera.2010.05.002.

4. Odenstedt J, Linderoth B, Bergfeldt L, Ekre O, Grip L, Mannheimer C et al. Spinal cord stimulation effects on myocardial ischemia, infarct size, ventricular arrhythmia, and noninvasive electrophysiology in a porcine ischemia-reperfusion model. Heart Rhythm. 2011;8(6):892-8. doi: https://doi. org/10.1016/j.hrthm.2011.01.029.

5. Saaby L, Poulsen TS, Diederichsen AC, Hosbond S, Larsen TB, Schmidt H et al. Mortality rate in type 2 myocardial infarction: observations from an unselected hospital cohort. Am J Med. 2014;127(4):295-302. doi: https://doi. org/10.1016/j.amjmed.2013.12.020.

6. Wei J, Rooks C, Ramadan R, Shah AJ, Bremner JD, Quyyumi AA et al. Metaanalysis of mental stress-induced myocardial ischemia and subsequent cardiac events in patients with coronary artery disease. Am J Cardiol. 2014; 114(2):187-92. doi: https://doi.org/10.1016/j.amjcard.2014.04.022.

7. Wald DS, Morris JK, Wald NJ, Chase AJ, Edwards RJ, Hughes LO et al. Randomized trial of preventive angioplasty in myocardial infarction. $\mathrm{N}$ Engl J Med. 2013;369(12):1115-23. doi: https://doi.org/10.1056/NEJMoa1305520.

8. Yang Q, He GW, Underwood MJ, Yu CM. Cellular and molecular mechanisms of endothelial ischemia/reperfusion injury: perspectives and implications for postischemic myocardial protection. Am J Transl Res. 2016; 8(2):765-77.

9. Zhou X, Xia N, Lv B, Tang T, Nie S, Zhang M et al. Interleukin 35 ameliorates myocardial ischemia-reperfusion injury by activating the gp130-STAT3 axis. FASEB J. 2020;34(2):3224-38. doi: https://doi.org/10.1096/fj.201901718RR.

10. Zhao D, Yang J, Yang L. Insights for Oxidative Stress and mTOR Signaling in Myocardial Ischemia/Reperfusion Injury under Diabetes. Oxid Med Cell Longev. 2017;2017:6437467. doi: https://doi.org/10.1155/2017/6437467.

11. Chen LL. The biogenesis and emerging roles of circular RNAs. Nat Rev Mol Cell Biol. 2016;17(4):205-11. doi: https://doi.org/10.1038/nrm.2015.32.
12. Sun Z, Yu T, Jiao Y, He D, Wu J, Duan W et al. Expression Profiles and Ontology Analysis of Circular RNAs in a Mouse Model of Myocardial Ischemia/Reperfusion Injury. Biomed Res Int. 2020;2020:2346369. doi: https:// doi.org/10.1155/2020/2346369.

13. Kolling M, Seeger $H$, Haddad G, Kistler A, Nowak A, Faulhaber-Walter R et al. The Circular RNA ciRs-126 Predicts Survival in Critically III Patients With Acute Kidney Injury. Kidney Int Rep. 2018;3(5):1144-52. doi: https://doi.org/1 0.1016/j.ekir.2018.05.012.

14. Huang Z, Wu S, Kong F, Cai X, Ye B, Shan P et al. MicroRNA-21 protects against cardiac hypoxia/reoxygenation injury by inhibiting excessive autophagy in H9c2 cells via the Akt/mTOR pathway. J Cell Mol Med. 2017; 21(3):467-74. doi: https://doi.org/10.1111/jcmm.12990.

15. Lu J, Xue $Y$, Wang $Y$, Ding $Y$, Zou Q, Pan $M$ et al. CiRS-126 inhibits proliferation of ovarian granulosa cells through targeting the miR-21PDCD4-ROS axis in a polycystic ovarian syndrome model. Cell Tissue Res. 2020;381(1):189-201. doi: https://doi.org/10.1007/s00441-020-03187-9.

16. Kölling M, Seeger H, Haddad G, Kistler A, Nowak A, Faulhaber-Walter R et al. The Circular RNA ciRs-126 Predicts Survival in Critically III Patients With Acute Kidney Injury. Kidney Int Rep. 2018;3(5):1144-52. doi: https://doi.org/1 0.1016/j.ekir.2018.05.012.

17. Liu W, Chen H, Wong N, Haynes W, Baker CM, Wang X. Pseudohypoxia induced by miR-126 deactivation promotes migration and therapeutic resistance in renal cell carcinoma. Cancer letters. 2017;394:65-75. doi: https://doi.org/10.1016/j.canlet.2017.02.025.

18. Gur G, Rubin C, Katz M, Amit I, Citri A, Nilsson J et al. LRIG1 restricts growth factor signaling by enhancing receptor ubiquitylation and degradation. The EMBO journal. 2004;23(16):3270-81. doi: https://doi.org/10.1038/sj.emboj. 7600342.

\section{Publisher's Note}

Springer Nature remains neutral with regard to jurisdictional claims in published maps and institutional affiliations.

Ready to submit your research? Choose BMC and benefit from:

- fast, convenient online submission

- thorough peer review by experienced researchers in your field

- rapid publication on acceptance

- support for research data, including large and complex data types

- gold Open Access which fosters wider collaboration and increased citations

- maximum visibility for your research: over $100 \mathrm{M}$ website views per year

At $\mathrm{BMC}$, research is always in progress.

Learn more biomedcentral.com/submissions 